Теорія Ймовір. та Матем. Статист. Вип. 80, 2009
Theor. Probability and Math. Statist.

No. 80, 2010, Pages 111-117

S 0094-9000(2010)00798-8

Article electronically published on August 19, 2010

\title{
THE LAW OF LARGE NUMBERS FOR THE MAX-SCHEME IN BANACH LATTICES
}

UDC 519.21

\author{
I. K. MATSAK
}

\begin{abstract}
We prove that the law of large numbers for the max-scheme in Banach lattices is equivalent to the condition that $\mathrm{E}\|X\|<\infty$. Some generalizations of this proposition are considered.
\end{abstract}

\section{Introduction. Main Results}

Let $B$ be a Banach space equipped with a norm $\|\cdot\|$ and let $X_{i}, i \geq 1$, be a sequence of independent copies of a random element $X$ with values in $B, S_{n}=\sum_{i=1}^{n} X_{i}$. It is known for the case of a separable Banach space $B$ that a random element $X$ satisfies the Kolmogorov law of large numbers

$$
\lim _{n \rightarrow \infty} \frac{\left\|S_{n}\right\|}{n}=0 \quad \text { a.s. }
$$

("a.s." is the abbreviation for "almost surely") if and only if

$$
\mathrm{E}\|X\|<\infty
$$

and $\mathrm{E} X=0$ (see, for example, [1]-[3]).

In what follows $B$ denotes a separable Banach lattice equipped with a module $|\cdot|$. Then the random element

$$
Z_{n}=\max _{1 \leq k \leq n}\left|X_{k}\right|
$$

is well defined in $B$ and one can study the following relation:

$$
\lim _{n \rightarrow \infty}\left\|\frac{Z_{n}}{n}\right\|=0 \quad \text { a.s. }
$$

We introduce a counterpart of the law of large numbers (11) for $Z_{n}$ by saying that a random element $X$ satisfies the law of large numbers for the max-scheme if relation (3) holds.

The order convergence in the law of large numbers for a sequence of independent random elements assuming values in Banach lattices is essentially different from the convergence in the norm [4. However, we show below that the law of large numbers (3) for the max-scheme is equivalent to the law of large numbers (1).

The following are the main results of this paper.

2000 Mathematics Subject Classification. Primary 60B12.

Key words and phrases. Max-scheme, Banach lattices, law of large numbers.

(C)2010 American Mathematical Society 
Theorem 1. Let $B$ be a separable Banach lattice and let $X$ be a random element assuming values in $B$. Then

(i) if condition (21) holds, then $X$ satisfies the law of large numbers (31) for the maxscheme;

(ii) if condition (2) does not hold, then

$$
\limsup _{n \rightarrow \infty}\left\|\frac{Z_{n}}{n}\right\|=\infty \quad \text { a.s. }
$$

Relation (31) can be improved if some extra assumptions are imposed on the Banach lattice.

Let $1 \leq p<\infty$. A Banach lattice $B$ is called $p$-convex if there exists a constant $D^{(p)}=D^{(p)}(B)$ such that

$$
\left\|\left(\sum_{i=1}^{n}\left|x_{i}\right|^{p}\right)^{1 / p}\right\| \leq D^{(p)}\left(\sum_{i=1}^{n}\left\|x_{i}\right\|^{p}\right)^{1 / p}
$$

for all $n$ and for all elements $\left(x_{i}\right)_{1}^{n} \subset B$. Similarly, $B$ is called $q$-concave if for some constant $D_{(q)}=D_{(q)}(B)$ the inverse inequality holds, namely,

$$
\left(\sum_{i=1}^{n}\left\|x_{i}\right\|^{q}\right)^{1 / q} \leq D_{(q)}\left\|\left(\sum_{i=1}^{n}\left|x_{i}\right|^{q}\right)^{1 / q}\right\| .
$$

Theorem 2. Let $B$ be a separable $p$-concave Banach lattice, $1<p<\infty$, and let $X$ be a random element assuming values in $B$. Then the following two conditions are equivalent:

$$
\begin{gathered}
\mathrm{E}\|X\|^{p}<\infty, \\
\lim _{n \rightarrow \infty}\left\|\frac{Z_{n}}{n^{1 / p}}\right\|=0 \quad \text { a.s. }
\end{gathered}
$$

Corollary 1. Let $X$ be a random element assuming values in the space $L_{p}\left(\ell_{p}\right)$ with $1<p<\infty$. Then conditions (5) and (6) of Theorem 2 are equivalent.

Remark 1. Theorem 2 does not hold for a general Banach lattice. We construct below an example of a random element assuming values in the space $\ell_{1}$ for which condition (5) holds for all $p \geq 1$, but the law of large numbers (6) is not satisfied.

Remark 2. It is shown in the paper 5 that

$$
\left|\frac{X_{n}}{n^{1 / p}}\right|
$$

almost surely converges to 0 in the sense of the order convergence for a separable $q$ concave Banach lattice, $1 \leq q<\infty$, if at least one of the following conditions is satisfied:

(i) $p<q$ and $\mathfrak{S}_{q}(X)$ exists;

(ii) $p=q, \phi(t)=|t|^{q} \ln \left(1+|t|^{q}\right)$ and $\mathfrak{S}_{\phi}(X)$ exists;

(iii) $p>q$ and $\mathfrak{S}_{p}(X)$ exists,

where $\mathfrak{S}_{q}(X)$ is the mean deviation of order $q$; for function spaces on a measurable space $(T, \Lambda, \mu)$, the mean deviation of order $q$ is defined by

$$
\mathfrak{S}_{q}(X)=\left(\left(\mathrm{E}|X(t)|^{q}\right)^{1 / q}, t \in T\right) .
$$

The definition of the mean deviations $\mathfrak{S}_{q}(X)$ and $\mathfrak{S}_{\phi}(X)$ for abstract Banach lattices can be found in [5].

The above remark implies that conditions (i)-(iii) are sufficient for the law of large numbers (6) in a $q$-concave Banach lattice. 


\section{Proofs}

Proof of Theorem 1. (i) Step 1. Assume that the values of the random element $X$ belong to a countable set of elements $\left(x_{1}, x_{2}, \ldots, x_{n}, \ldots\right)$. Then we represent $X$ as follows:

$$
X=\sum_{i=1}^{\infty} \theta_{i} x_{i}
$$

where

$$
\sum_{i=1}^{\infty} \theta_{i}=1, \quad \mathrm{P}\left(\theta_{i}=1\right)=p_{i}, \quad \mathrm{P}\left(\theta_{i}=0\right)=1-p_{i} .
$$

By condition (2),

$$
\mathrm{E}\|X\|=\sum_{i=1}^{\infty} p_{i}\left\|x_{i}\right\|<\infty
$$

Let $X_{n}=\sum_{i=1}^{\infty} \theta_{n i} x_{i}, n \geq 1$, be independent copies of $X$. Fix a positive integer $k$ and represent $X_{n}$ as follows:

$$
\begin{gathered}
X_{n}=X_{n}^{(k)}+R_{n}^{(k)}, \\
X_{n}^{(k)}=\sum_{i=1}^{k} \theta_{n i} x_{i}, \quad R_{n}^{(k)}=\sum_{i=k+1}^{\infty} \theta_{n i} x_{i} .
\end{gathered}
$$

It is clear that

$$
\left|\frac{Z_{n}}{n}\right| \leq \frac{1}{n} \sup _{1 \leq j \leq n}\left|X_{j}^{(k)}\right|+\frac{1}{n} \sup _{1 \leq j \leq n}\left|R_{j}^{(k)}\right| \quad \text { a.s. }
$$

Since $0 \leq \theta_{j i} \leq 1$, we have

$$
\left\|\frac{1}{n} \sup _{1 \leq j \leq n}\left|X_{j}^{(k)}\right|\right\| \leq \sum_{i=1}^{k} \frac{\sup _{1 \leq j \leq n} \theta_{j i}}{n}\left\|x_{i}\right\| \rightarrow 0 \quad \text { a.s. }
$$

as $n \rightarrow \infty$.

We estimate the second term on the right hand side of inequality (8) in the following way:

$$
\left\|\frac{1}{n} \sup _{1 \leq j \leq n}\left|R_{j}^{(k)}\right|\right\| \leq \frac{1}{n}\left\|\sum_{j=1}^{n}\left|R_{j}^{(k)}\right|\right\| \leq \frac{1}{n} \sum_{j=1}^{n}\left\|R_{j}^{(k)}\right\| .
$$

According to the law of large numbers in $\mathbf{R}^{1}$,

$$
\frac{1}{n} \sum_{j=1}^{n}\left\|R_{j}^{(k)}\right\| \rightarrow \mathrm{E}\left\|R_{1}^{(k)}\right\| \quad \text { a.s. }
$$

By condition (7), for a given $\varepsilon>0$ there exists a number $k_{0}$ such that

$$
\mathrm{E}\left\|R_{1}^{(k)}\right\|=\sum_{i=k+1}^{\infty} p_{i}\left\|x_{i}\right\|<\varepsilon
$$

for all $k>k_{0}$. It remains to pass to the limit in (8) as $n \rightarrow \infty$ and then as $k \rightarrow \infty$. This together with the bounds (9)-(11) completes the proof of (3).

Step 2. Consider a general case. In the separable Banach space $B$, there exists an everywhere dense in $B$ sequence $\left(x_{1}, x_{2}, \ldots, x_{n}, \ldots\right)$. Fix an arbitrary number $\varepsilon>0$ and denote by

$$
U_{i}(\varepsilon)=\left\{x \in B:\left\|x-x_{i}\right\|<\varepsilon\right\}
$$


the ball of radius $\varepsilon$ centered at the point $x_{i}$. Further let

$$
\begin{gathered}
V_{1}(\varepsilon)=U_{1}(\varepsilon), \\
V_{2}(\varepsilon)=U_{2}(\varepsilon) \cap \bar{U}_{1}(\varepsilon), \\
\ldots \\
V_{n}(\varepsilon)=U_{n}(\varepsilon) \bigcap_{i=1}^{n-1} \bar{U}_{i}(\varepsilon), \\
\ldots
\end{gathered}
$$

where $\bar{A}$ is the complement of the set $A$. Then

$$
\bigcup_{i=1}^{\infty} V_{i}(\varepsilon)=B
$$

Moreover, if $i \neq j$, then

$$
V_{i}(\varepsilon) \cap V_{j}(\varepsilon)=\varnothing .
$$

Let $T_{\varepsilon}$ be an operator acting in $B$ and such that

$$
T_{\varepsilon}(x)=x_{i}
$$

if $x \in V_{i}(\varepsilon)$. It is clear that the sequence of random elements $\left(T_{\varepsilon}\left(X_{n}\right)\right)$ satisfies the conditions used in Step 1. Thus

$$
\lim _{n \rightarrow \infty}\left\|\frac{\sup _{1 \leq j \leq n}\left|T_{\varepsilon}\left(X_{j}\right)\right|}{n}\right\|=0 \quad \text { a.s. }
$$

Further we use the following elementary inequality:

$$
\left|\max _{1 \leq i \leq n}\right| a_{i}\left|-\max _{1 \leq i \leq n}\right| b_{i}|| \leq \max _{1 \leq i \leq n}\left|a_{i}-b_{i}\right| .
$$

Hence

$$
\left|Z_{n}-\sup _{1 \leq j \leq n}\right| T_{\varepsilon}\left(X_{j}\right)|| \leq \sup _{1 \leq j \leq n}\left|X_{j}-T_{\varepsilon}\left(X_{j}\right)\right| \leq \sum_{j=1}^{n}\left|X_{j}-T_{\varepsilon}\left(X_{j}\right)\right|,
$$

whence

$$
\frac{1}{n}\left\|Z_{n}-\sup _{1 \leq j \leq n}\left|T_{\varepsilon}\left(X_{j}\right)\right|\right\| \leq \frac{1}{n} \sum_{j=1}^{n}\left\|X_{j}-T_{\varepsilon}\left(X_{j}\right)\right\| \leq \varepsilon,
$$

since

$$
\left\|T_{\varepsilon}(x)-x\right\| \leq \varepsilon
$$

for all $x \in B$ by the construction of the operator $T_{\varepsilon}$.

Since the number $\varepsilon>0$ is arbitrary, the latter bounds together with relation (12) prove the law of large numbers (3).

Note that the reasoning presented above is close to the classical proof of the law of large numbers for Banach spaces.

(ii). Let $\mathrm{E}\|X\|=\infty$. Assume that

$$
\limsup _{n \rightarrow \infty} \frac{\left\|X_{n}\right\|}{n} \leq \limsup _{n \rightarrow \infty} \frac{\left\|Z_{n}\right\|}{n}=C_{1}<\infty \quad \text { a.s. }
$$

Then only a finite number of random events $A_{n}=\left\{\left\|X_{n}\right\| \geq C n\right\}$ occur almost surely if $C>C_{1}$. Thus the Borel-Cantelli lemma implies that

$$
\sum_{n=1}^{\infty} \mathrm{P}\left(A_{n}\right)<\infty
$$


On the other hand, if $F(x)$ denotes the distribution function of the random variable $\left\|X_{n}\right\|$, then

whence

$$
\mathrm{P}\left(A_{n}\right) \geq 1-F(C n)
$$

$$
\begin{aligned}
\sum_{n=1}^{\infty} \mathrm{P}\left(A_{n}\right) & \geq \sum_{n=1}^{\infty}(1-F(C n)) \geq \int_{C}^{\infty}(1-F(x)) d x \\
& \geq \int_{0}^{\infty}(1-F(x)) d x-C=\infty
\end{aligned}
$$

This contradicts inequality (13).

Proof of Theorem 2, Let condition (6) hold. Then

$$
\lim _{n \rightarrow \infty} \frac{\eta_{n}}{n}=\lim _{n \rightarrow \infty}\left\|\frac{X_{n}}{n^{1 / p}}\right\|=0 \quad \text { a.s. }
$$

where $\eta_{n}=\left\|X_{n}\right\|^{p}$. Following the reasoning of item (ii) in Theorem 1 we obtain from the latter relation that

$$
\mathrm{E} \eta_{n}=\mathrm{E}\left\|X_{n}\right\|^{p}<\infty
$$

The proof of the implication (5) $\Rightarrow(6)$ is the same as the proof of item (i) of Theorem(1. The only difference is that now we use the following bound:

$$
\left\|\frac{Z_{n}}{n^{1 / p}}\right\| \leq\left\|\left(\frac{1}{n} \sum_{i=1}^{n}\left|X_{i}\right|^{p}\right)^{1 / p}\right\| \leq D^{(p)}\left(\frac{1}{n} \sum_{i=1}^{n}\left\|X_{i}\right\|^{p}\right)^{1 / p} \quad \text { a.s. }
$$

which immediately follows from the $p$-concavity of the Banach lattice $B$.

\section{ExAmple}

A counterexample for the ordinal law of large numbers in Banach lattices is constructed in the paper 4. It turns out that the same random element $X$ can be used to show that the law of large numbers (6) does not hold. At the same time, condition (5) holds for all $p \geq 1$ for this random element $X$.

We consider a random element $X$ assuming values in the space $\ell_{1}$. Put $L(t)=\ln t$ for $t>2$ and $L(t)=1$ for $t \leq 2$,

$$
\theta=\sum_{k \geq 1} \frac{1}{k L^{2}(k)}, \quad p_{k}=\frac{1}{\theta k L^{2}(k)}, \quad k \geq 1 .
$$

It is clear that $\sum_{k \geq 1} p_{k}=1$.

Let $\left(\xi_{k}\right)$ be a sequence of independent random variables such that

$$
\mathrm{P}\left(\xi_{k}=+1\right)=p_{k} / 2, \quad \mathrm{P}\left(\xi_{k}=-1\right)=p_{k} / 2, \quad \mathrm{P}\left(\xi_{k}=0\right)=1-p_{k} .
$$

It is clear that only a finite number of components of the random element $X=\left(\xi_{k}\right)$ are nonzero almost surely. Therefore $X \in \ell_{1}$ and

$$
\mathrm{E}\left|\xi_{k}\right|=p_{k}, \quad \mathrm{E}\|X\|_{\ell_{1}}=\sum_{k \geq 1} p_{k}=1 .
$$

It is proved in 4 that the random element $X$ satisfies condition (5) for all $p \geq 1$.

Further we show that equality (6) does not hold for this random element. Let

$$
X_{n}=\left(\xi_{n k}\right),
$$

where $\left(\xi_{n k}\right)$ are independent copies of the sequence $\left(\xi_{k}\right)$. Put

$$
Y_{n}=\left\|\sup _{1 \leq m \leq n}\left|X_{m}\right|\right\|_{l_{1}}=\sum_{k \geq 1}\left|\kappa_{n k}\right|
$$


where $\kappa_{n k}=\sup _{1 \leq m \leq n}\left|\xi_{m k}\right|$ and

$$
\mathrm{P}\left(\kappa_{n k}=1\right)=b_{n k}, \quad \mathrm{P}\left(\kappa_{n k}=0\right)=1-b_{n k}, \quad b_{n k}=1-\left(1-p_{k}\right)^{n} .
$$

The series on the right hand side of equality (15) converges almost surely. Thus one can evaluate the first two moments of the random variable $Y_{n}$ :

$$
\begin{gathered}
\mathrm{E} Y_{n}=m_{n}=\sum_{k=1}^{\infty} b_{n k}, \\
\mathrm{E} Y_{n}^{2}=\sum_{k=1}^{\infty} b_{n k}+\sum_{k \neq j} b_{n k} b_{n j}=m_{n}^{2}+\sum_{k=1}^{\infty} b_{n k}\left(1-b_{n k}\right) \leq m_{n}^{2}+m_{n} .
\end{gathered}
$$

It is clear that $m_{n} \rightarrow \infty$.

Now we prove the following asymptotic lower bound for $m_{n}$ :

$$
\liminf _{n \rightarrow \infty} \frac{m_{n} \ln n}{n} \geq C>0 .
$$

First we check that the latter relations contradict the law of large numbers (6). Indeed, substituting $\lambda=\frac{1}{2}$ and $\zeta=Y_{n}$ in the known inequality (see Inequality II in Chapter I, $\S 6$ of [6])

$$
\mathrm{P}(\zeta \geq \lambda \mathrm{E} \zeta) \geq(1-\lambda)^{2} \frac{(\mathrm{E} \zeta)^{2}}{\mathrm{E} \zeta^{2}}
$$

and choosing $0<C_{1}<C$ (the constant $C$ is defined by the asymptotic lower bound (17)) we obtain

$$
\mathrm{P}\left(\frac{Y_{n} \ln n}{n} \geq \frac{C_{1}}{2}\right) \geq \mathrm{P}\left(Y_{n} \geq \frac{1}{2} \mathrm{E} Y_{n}\right) \geq \frac{\left(\mathrm{E} Y_{n}\right)^{2}}{4 \mathrm{E} Y_{n}^{2}}
$$

for sufficiently large $n$. This together with (16) implies the inequality

$$
\mathrm{P}\left(\frac{Y_{n} \ln n}{n} \geq \frac{C_{1}}{2}\right) \geq \frac{1}{4+4 / m_{n}} \geq \frac{1}{8},
$$

which contradicts the law of large numbers (6).

It remains to prove the lower bound (17). We need the following known elementary inequality (see Chapter II, $\S 15$ of [7]):

$$
x^{r}-y^{r} \geq r y^{r-1}(x-y)
$$

for all $x>0, y>0$, and $r \geq 1$. Let $x=1, y=1-p_{k}$, and $r=n$. Then

$$
b_{n k} \geq n p_{k}\left(1-p_{k}\right)^{n-1} \text {. }
$$

Further,

$$
\begin{aligned}
m_{n} & \geq n \sum_{k \geq 1} p_{k}\left(1-p_{k}\right)^{n-1}=\frac{n}{\theta} \sum_{k \geq 1} \frac{1}{k L^{2}(k)}\left(1-\frac{1}{\theta k L^{2}(k)}\right)^{n-1} \\
& \geq \frac{n}{\theta}\left(1-\frac{1}{\theta n L^{2}(n)}\right)^{n-1} \sum_{k \geq n} \frac{1}{k L^{2}(k)} .
\end{aligned}
$$

As $n \rightarrow \infty$, we have

$$
\begin{aligned}
& \sum_{k \geq n} \frac{1}{k \ln ^{2}(k)} \sim \int_{n}^{\infty} \frac{d x}{x \ln ^{2}(x)} \sim \frac{1}{\ln n}, \quad \text { and } \\
& \left(1-\frac{1}{\theta n \ln ^{2}(n)}\right)^{n} \sim \exp \left(-\frac{1}{\theta \ln ^{2}(n)}\right) \rightarrow 1 .
\end{aligned}
$$

Combining together bounds (18)-(20) we get the asymptotic lower bound (17). 


\section{BIBLIOGRAPHY}

1. W. Feller, An Introduction to Probability Theory and Its Applications, vol. II, John Wiley \& Sons, Inc., New York-London-Sydney, 1971. MR0270403 (42:5292)

2. E. Mourier, Eléments aléatoires dans un espace de Banach, Ann. Inst. H. Poincaré 19 (1953), 161-244. MR0064339 (16:268a)

3. M. Ledoux and M. Talagrand, Probability in Banach Spaces, Springer, Berlin, 1991. MR.1102015 (93c:60001)

4. I. K. Matsak, Some remarks on the ordinal strong law of large numbers, Teor. Imovirnost. Matem. Statist. 72 (2005), 84-92; English transl. in Theory Probab. Math. Statist. 72 (2006), 93-102. MR2168139 (2006f:60011)

5. I. K. Matsak, Estimates for the moments of the supremum of normalized sums of independent random variables, Teor. Imovirnost. Matem. Statist. 67 (2002), 104-116; English transl. in Theory Probab. Math. Statist. 67 (2003), 115-128. MR.1956624 (2004i:60061)

6. J.-P. Kahane, Some Random Series of Functions, D. C. Heath and Company, Lexington, Massachusetts, 1968. MR0254888(40:8095)

7. G. Hardy, J. E. Littlewood, and G. Pólya, Inequalities, 2nd ed., Cambridge University Press, Cambridge, UK, 1952. MR0046395(13:727e)

Department of Operations Research, Faculty for Cybernetics, National taras Shevchenko University, Academician Glushkov Avenue, 6, Kiev 03127, Ukraine

E-mail address: mik@unicyb.kiev.ua

Received 10/APR/2008

Translated by N. N. SEMENOV 